\title{
Estudo e Simulação do Processo de Adoçamento de Gás Natural
}

\author{
A. F. B. Ferreira ${ }^{1}$ e N. S. Amorim² \\ ${ }^{1}$ Universidade do Estado do Amapá, Colegiado de Engenharia Química \\ ${ }^{2}$ Universidade Federal do Pará, Colegiado de Engenharia Química \\ E-mail para contato: ailtonfreitas_ap@hotmail.com
}

\begin{abstract}
RESUMO - O gás natural apresenta alguns contaminantes, como o ácido sulfídrico $\left(\mathrm{H}_{2} \mathrm{~S}\right)$ e o dióxido de carbono $\left(\mathrm{CO}_{2}\right)$, que devem ser removidos por serem tóxicos, corrosivos e prejudiciais as tubulações que transportam o gás. Um dos processos mais utilizados para a eliminação dessas substâncias é o adoçamento, que remove esses gases ácidos mediante o uso de alcanolaminas, tais como a DEA. Com o avanço tecnológico, este processo pode ser avaliado através do simulador ASPEN PLUS, o diferencial deste consiste em poder usá-lo sob diferentes condições de operações. Portanto, o presente trabalho tem como objetivo avaliar o comportamento de uma etapa do tratamento de gás natural, através do simulador de processos químicos ASPEN PLUS ${ }^{\circledR} 2006$, variando as condições de operação em relação a temperatura e a concentração da solução aquosa de DEA, simulando primeiro no absorvedor e posteriormente o processo em reciclo (regeneração).
\end{abstract}

Palavras-chave: gás natural, adoçamento, gases ácidos, DEA, ASPEN PLUS.

\section{INTRODUÇÃO}

Tavalera (2002) argumenta que plantas de processamento de gás natural são projetadas para remover ou reduzir certos componentes indesejáveis, como o $\mathrm{H}_{2} \mathrm{~S}$ e $\mathrm{CO}_{2}$. A remoção desses contaminantes é fundamental para produzir um gás que atenda as especificações de mercado, sem mencionar também que esses gases são tóxicos e podem provocar a corrosão e o entupimento das válvulas que transportam o gás. Sendo assim e, com o atual avanço computacional, verifica-se que a simulação de processos químicos é importante, pois os resultados obtidos com as simulações computacionais podem auxiliar no processo real e representar diversas etapas e, consequentemente, antecipar o comportamento delas.

\section{Metodologia}

O processo foi simulado em regime permanente, sem usar o método da eficiência e tendo a DEA como solvente, pois, segundo Coelho (2007), esta amina é a menos corrosiva em concentrações acima de $20 \%$ e sem a eficiência, porque não se considerou a perda de carga nem a transferência de massa e de calor. Inicialmente, foram avaliadas as frações de $\mathrm{H}_{2} \mathrm{~S}$ e $\mathrm{CO}_{2}$ que saem do absorvedor sem reciclo e, posteriormente, aquelas que saem no reciclo, variando a temperatura de entrada na corrente da amina e as concentrações de DEA, com o objetivo de verificar a influência desses parâmetros no processo. Na regeneração, usou-se do método de estágios em equilíbrio. Além disso, o modelo termodinâmico do equilíbrio líquidovapor escolhido foi o de WILSON. Por fim, este modelo foi comparado com dados da 
literatura para determinar o Load (número de moles do gás ácido absorvido na fase líquida pelo número de moles da amina).

\subsection{Composições e condições usadas nas simulações}

A composição do gás natural foi obtida a partir do trabalho de Mendieta (2011), sendo apresentada na tabela 1. Já as condições operacionais das simulações no absorvedor, em relação as correntes de gás natural e de amina, estão disponíveis na tabela 2.

Tabela 1 - Fração molar.

\begin{tabular}{ccccccccc}
\hline Componentes & Etano & Propano & Butano & Propenos & Butenos & Pentano & Argônio \\
\hline Gás Natural & 0,1167 & 0,013 & 0,0008 & 0,0566 & 0,002 & 0,004 & 0,0003 \\
\hline Componentes & $\mathrm{H}_{2} \mathrm{O}$ & $\mathrm{CO}_{2}$ & $\mathrm{H}_{2} \mathrm{~S}$ & DEA & $\mathrm{CH}_{4}$ & $\mathrm{~N}_{2}$ & $\mathrm{H}_{2}$ & $\mathrm{CO}$ \\
\hline Gás Natural & 0 & 0,0183 & 0,0254 & 0 & 0,3669 & 0,0516 & 0,2334 & 0,0084
\end{tabular}

Tabela 2 - Condições iniciais para o gás natural e para a amina no absorvedor.

\begin{tabular}{ccc}
\hline Condição Operacional & Corrente do Gás Natural & Corrente de Amina Pobre \\
\hline Temperatura $\left({ }^{\circ} \mathrm{C}\right)$ & 22 & 27 \\
Pressão $(\mathrm{atm})$ & 69 & 69 \\
Vazão $(\mathrm{Kg} / \mathrm{h})$ & 25000 & 40000 \\
\hline
\end{tabular}

A etapa de regeneração da amina necessita de alguns parâmetros, de acordo com Coelho (2007), e de uma composição do gás no regenerador, esta última foi obtida a partir da corrente de fundo do absorvedor. As tabelas 3 e 4 a seguir mostram essas propriedades e a composição para a regeneração.

Tabela 3 - Parâmetros e propriedades para o regenerador.

\begin{tabular}{cc}
\hline Parâmetro & Valor/Condição \\
\hline Carga Térmica no Condensador & $-3 \mathrm{MMKcal} / \mathrm{h}$ \\
Razão de Refluxo (RR) & 1 \\
\hline Propriedades & Valores \\
\hline Vazão & $43163,75 \mathrm{Kg} / \mathrm{h}$ \\
Pressão & $5,886 \mathrm{~atm}$ \\
Temperatura & $95^{\circ} \mathrm{C}$ \\
\hline
\end{tabular}



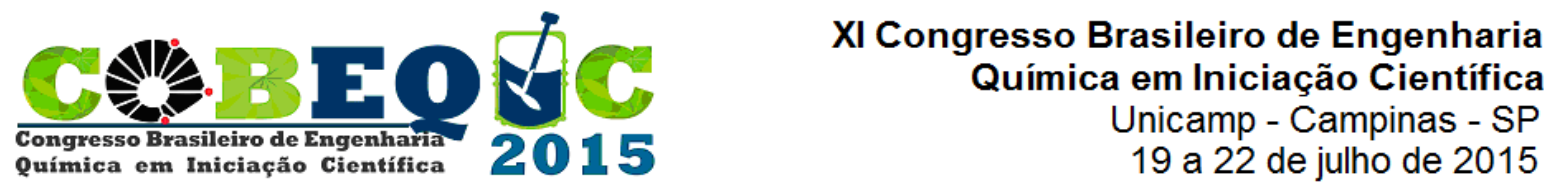

Tabela 4 - Corrente de alimentação do regenerador.

\begin{tabular}{|c|c|c|c|c|c|c|c|}
\hline Componentes & Etano & Propano & Butano & Propenos & Butenos & Pentano & Argônio \\
\hline $\begin{array}{l}\text { Fração } \\
\text { Molar }\end{array}$ & $1,9 \mathrm{E}^{-10}$ & $1,02 \mathrm{E}^{-13}$ & $4,29 \mathrm{E}^{-13}$ & $1,07 \mathrm{E}^{-11}$ & $2,1 \mathrm{E}^{-13}$ & $8,47 \mathrm{E}^{-15}$ & $1,47 \mathrm{E}^{-12}$ \\
\hline Componentes & $\mathrm{H}_{2} \mathrm{O}$ & $\mathrm{CO}_{2}$ & $\mathrm{H}_{2} \mathrm{~S}$ & DEA & $\mathrm{CH}_{4}$ & $\mathrm{~N}_{2}$ & $\mathrm{CO}$ \\
\hline $\begin{array}{l}\text { Fração } \\
\text { Molar }\end{array}$ & 0 & 0,0003 & 0,000475 & 0 & $1,33 \mathrm{E}^{-8}$ & $2,76 \mathrm{E}^{-9}$ & $2,75 \mathrm{E}-8$ \\
\hline
\end{tabular}

\subsection{Variáveis analisadas nas simulações}

Os parâmetros para o absorvedor e regenerador estão apresentados na tabela 5 abaixo.

Tabela 5 - Parâmetros importantes para o absorvedor e regenerador.

\begin{tabular}{ccc}
\hline Parâmetros avaliados & Faixa (intervalo) & Valores usados nas simulações \\
\hline Temperatura $\left({ }^{\circ} \mathrm{C}\right)$ & $25-45$ & $27,32,37$ e 42 \\
Concentração de DEA $(\%)$ & $20-40$ & $25,30,35$ e 40 \\
Concentração de $\mathrm{H}_{2} \mathrm{O}(\%)$ & $60-75$ & $75,70,65$ e 60 \\
Prato de alimentação & $2-8$ & 3 \\
Temperatura de regeneração $\left({ }^{\circ} \mathrm{C}\right)$ & $75-115$ & 95 \\
RR & $1-75$ & 1 \\
Número de estágios & $6-20$ & 9 \\
\hline
\end{tabular}

\section{RESULTADOS}

\subsection{Simulação no Absorvedor (sem reciclo)}

Na simulação do absorvedor, os dados obtidos estão descritos nas tabelas 6 e 7 a seguir.

Tabela 6 - Variação da temperatura na corrente de amina pobre.

\begin{tabular}{ccccc}
\hline $\begin{array}{c}\text { Temperatura da } \\
\text { corrente amina } \\
\text { pobre }\left({ }^{\circ} \mathrm{C}\right)\end{array}$ & $\begin{array}{c}\text { Vazão de } \mathrm{CO}_{2} \\
\text { na corrente de } \\
\text { gás doce }(\mathrm{Kg} / \mathrm{h})\end{array}$ & $\begin{array}{c}\text { Fração molar } \\
\text { de } \mathrm{CO}_{2} \\
(\text { gás doce) }\end{array}$ & $\begin{array}{c}\text { Vazão de } \mathrm{H}_{2} \mathrm{~S} \\
\text { na corrente de } \\
\text { gás doce }(\mathrm{Kg} / \mathrm{h})\end{array}$ & $\begin{array}{c}\text { Fração molar } \\
\text { de } \mathrm{H}_{2} \mathrm{~S} \\
\text { (gás doce })\end{array}$ \\
\hline 27 & 0,0979 & $2,9858 \times 10^{-6}$ & $1,3692 \times 10^{-6}$ & $5,3904 \times 10^{-11}$ \\
32 & 0,2577 & $7,7383 \times 10^{-6}$ & $4,0723 \times 10^{-6}$ & $1,5786 \times 10^{-10}$ \\
37 & 0,6779 & $2,0053 \times 10^{-5}$ & $1,9363 \times 10^{-5}$ & $4,6902 \times 10^{-10}$ \\
42 & 1,8517 & $5,417 \times 10^{-5}$ & $3,9852 \times 10^{-5}$ & $1,5054 \times 10^{-9}$ \\
\hline
\end{tabular}


Tabela 7 - Variação da concentração de DEA (\%) na corrente de amina pobre.

\begin{tabular}{ccccc}
\hline $\begin{array}{c}\text { Concentração } \\
\text { de DEA } \\
(\%)\end{array}$ & $\begin{array}{c}\text { Vazão de } \mathrm{CO}_{2} \\
\text { na corrente de } \\
\text { gás doce }(\mathrm{Kg} / \mathrm{h})\end{array}$ & $\begin{array}{c}\text { Fração molar } \\
\text { de } \mathrm{CO}_{2} \\
(\text { gás doce) }\end{array}$ & $\begin{array}{c}\text { Vazão de } \mathrm{H}_{2} \mathrm{~S} \\
\text { na corrente de } \\
\text { gás doce }(\mathrm{Kg} / \mathrm{h})\end{array}$ & $\begin{array}{c}\text { Fração molar } \\
\text { de } \mathrm{H}_{2} \mathrm{~S} \\
(\text { gás doce })\end{array}$ \\
\hline 25 & 0,0979 & $2,9858 \times 10^{-6}$ & $1,3692 \times 10^{-6}$ & $5,3904 \times 10^{-11}$ \\
30 & 0,1213 & $3,6664 \times 10^{-6}$ & $1,7164 \times 10^{-6}$ & $6,6982 \times 10^{-11}$ \\
35 & 0,1578 & $4,7235 \times 10^{-6}$ & $2,2674 \times 10^{-6}$ & $8,7597 \times 10^{-11}$ \\
40 & 0,2115 & $6,2632 \times 10^{-6}$ & $3,0907 \times 10^{-6}$ & $1,1815 \times 10^{-10}$ \\
\hline
\end{tabular}

As figuras 1 e 2 abaixo mostram o comportamento das variações realizadas no processo.

Figura 1 - Variação da temperatura.

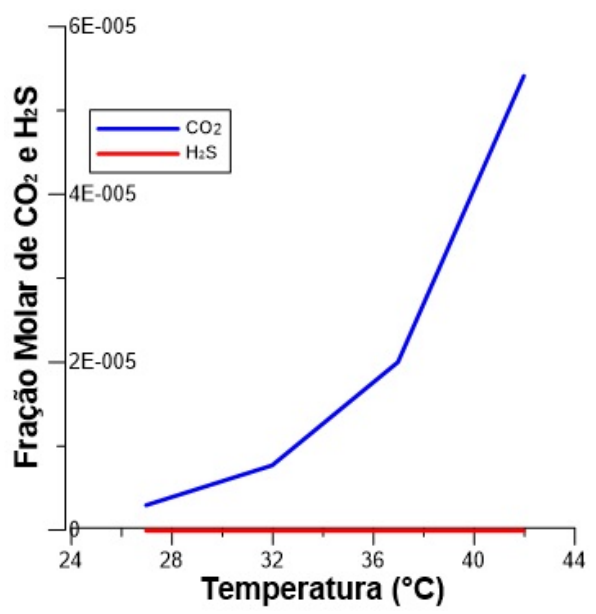

Figura 2 - Variação da concentração de DEA.

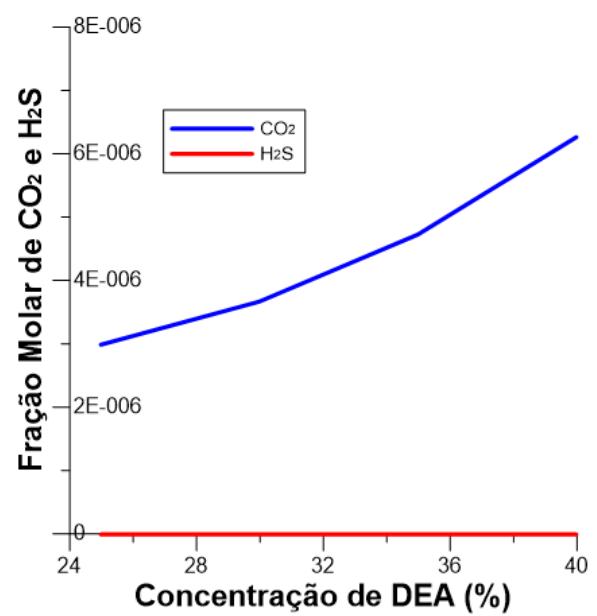

Verificando-se os gráficos, percebe-se que as frações molares dos ácidos aumentam mais com a variação da temperatura do que com a variação da concentração, logo, a temperatura favorece uma eliminação maior dessas substâncias na corrente de gás doce e piora a absorção dos componentes na corrente da amina, pois os mesmos estão em maior proporção no topo do absorvedor. Tanto o aumento da temperatura como o da concentração implicou numa retirada mais de $\mathrm{CO}_{2}$ do que de $\mathrm{H}_{2} \mathrm{~S}$, segundo Coelho (2007) este fato ocorre pelo não uso da eficiência nas simulações, pois na prática deseja-se eliminar mais $\mathrm{H}_{2} \mathrm{~S}$. Além disso, os dados obtidos no gráfico 2 não possuem o mesmo perfil da literatura, baseado no trabalho de Talavera (2002), visto que as frações molares deveriam diminuir com o aumento da concentração de DEA, então, é aconselhável usar concentrações menores da amina, considerar a eficiência nas simulações ou mudar o modelo termodinâmico para se ter um resultado melhor no absorvedor.

\subsection{Simulação com Reciclo}


As tabelas 9 e 10 a seguir apresentam as variações da temperatura e do percentual de DEA na etapa de regeneração.

Tabela 9 - Variação da temperatura na corrente de amina pobre.

\begin{tabular}{ccccc}
\hline $\begin{array}{c}\text { Temperatura da } \\
\begin{array}{c}\text { corrente amina } \\
\text { pobre }\left({ }^{\circ} \mathrm{C}\right)\end{array}\end{array}$ & $\begin{array}{c}\text { Vazão de } \mathrm{CO}_{2} \\
\text { na corrente de } \\
\text { gás doce }(\mathrm{Kg} / \mathrm{h})\end{array}$ & $\begin{array}{c}\text { Fração molar } \\
\text { de } \mathrm{CO}_{2} \\
(\text { Gás doce })\end{array}$ & $\begin{array}{c}\text { Vazão de } \mathrm{H}_{2} \mathrm{~S} \\
\text { na corrente de } \\
\text { gás doce }(\mathrm{Kg} / \mathrm{h})\end{array}$ & $\begin{array}{c}\text { Fração molar } \\
\text { de } \mathrm{H}_{2} \mathrm{~S} \\
(\text { Gás doce })\end{array}$ \\
\hline 27 & 0,05 & 0 & 0 & 0 \\
32 & 0,06 & 0 & 0 & 0 \\
37 & 0,31 & 0 & 0 & 0 \\
42 & 0,78 & 0 & 0 & 0 \\
\hline
\end{tabular}

Tabela 10 - Variação do percentual (\%) de DEA na corrente de amina pobre.

\begin{tabular}{ccccc}
\hline $\begin{array}{c}\text { Concentração } \\
\text { de DEA (\%) }\end{array}$ & $\begin{array}{c}\text { Vazão de } \mathrm{CO}_{2} \\
\text { na corrente de } \\
\text { gás doce }(\mathrm{Kg} / \mathrm{h})\end{array}$ & $\begin{array}{c}\text { Fração molar } \\
\text { de } \mathrm{CO}_{2} \\
(\text { Gás doce })\end{array}$ & $\begin{array}{c}\text { Vazão de } \mathrm{H}_{2} \mathrm{~S} \\
\text { na corrente de } \\
\text { gás doce }(\mathrm{Kg} / \mathrm{h})\end{array}$ & $\begin{array}{c}\text { Fração molar } \\
\text { de } \mathrm{H}_{2} \mathrm{~S} \\
(\mathrm{Gás} \text { doce })\end{array}$ \\
\hline 25 & 0,05 & 0 & 0 & 0 \\
30 & 0,06 & 0 & 0 & 0 \\
35 & 0,8 & 0 & 0 & 0 \\
40 & 0,1 & 0 & 0 & 0 \\
\hline
\end{tabular}

O reciclo é mais eficiente do que a simulação apenas no absorvedor, porque ocorreu a absorção total das frações de $\mathrm{H}_{2} \mathrm{~S}$ e $\mathrm{CO}_{2}$ em praticamente todas as correntes do processo; ressaltando que a variação do percentual de DEA aumentou as frações de $\mathrm{CO}_{2}$, indicando que para se ter um bom resultado é melhor trabalhar com baixas concentrações de DEA.

\subsection{Comparação das curvas de Load em função da Pressão Parcial}

As propriedades puras dos componentes do gás natural, geradas nas simulações sob diferentes pressões, estão representadas através da figura 3 e da figura 4 a seguir.

Figura 3 - Sistema DEA-CO . $^{2}$ 

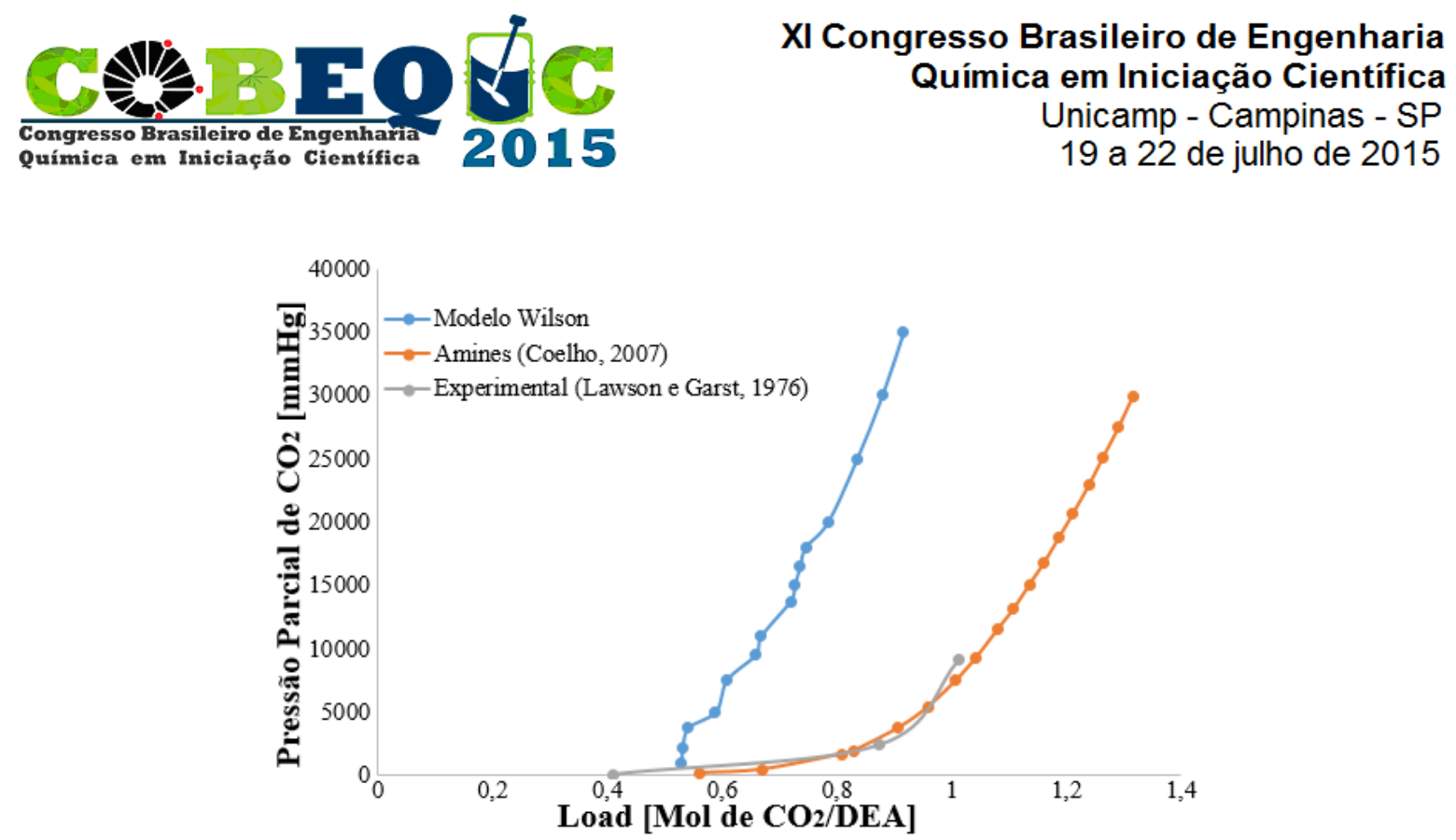

Figura 4 - Sistema DEA- $\mathrm{H}_{2} \mathrm{~S}$.

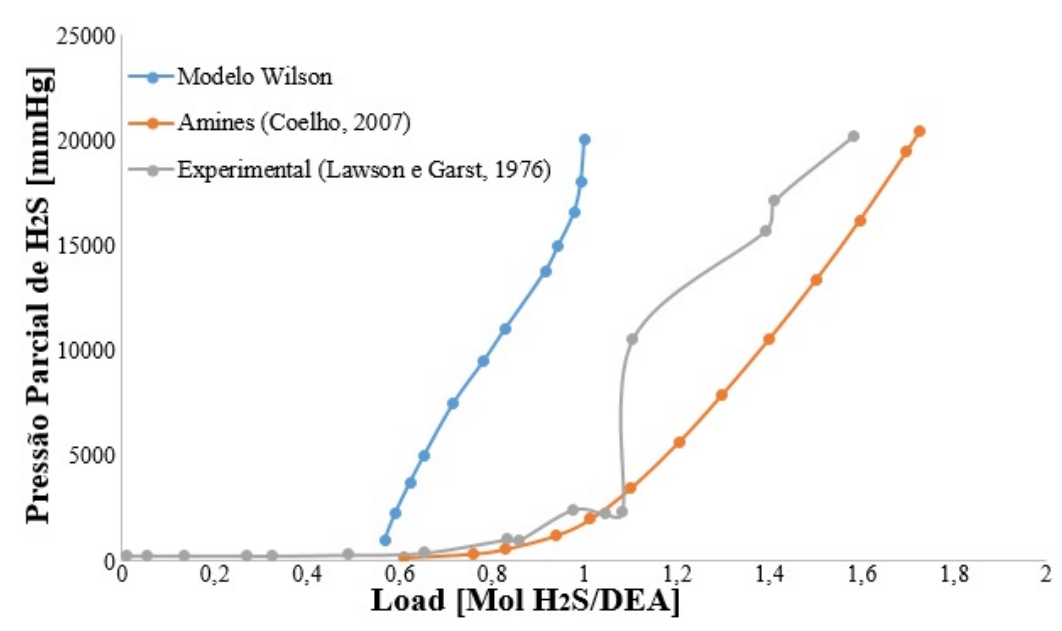

Analisando-se os gráficos, observa-se que os valores do Load são baixos para uma mesma pressão, indicando que ocorreu uma subestimação na absorção dos gases. Verifica-se também que a subestimação dos dados é maior para o $\mathrm{CO}_{2}$ do que para o $\mathrm{H}_{2} \mathrm{~S}$, diferentemente das suas pressões parciais na fase líquida, visto que estas estão mais elevadas do que as pressões do $\mathrm{H}_{2} \mathrm{~S}$.

\section{CONCLUSÃO}

Os resultados obtidos indicaram que o ASPEN PLUS é uma boa ferramenta no auxílio de uma tomada de decisão de projetos e na otimização de uma etapa do tratamento de gás natural, assim como a amina DEA, mas deve-se ter o cuidado na escolha do modelo termodinâmico, pois verificou-se que o modelo WILSON subestimou as quantidades absorvidas, por isso as frações molares apresentadas na corrente de gás doce foram baixas para o Load, da mesma forma que ele influenciou nas variações da temperatura e nas concentrações de amina. Portanto, recomenda-se que em outras simulações o modelo termodinâmico do equilíbrio líquido-vapor venha a ser modificado, pois este é importante na análise dos parâmetros a serem avaliados. 


\section{Congresso Brasileiro de Engenharia \\ Química em Iniciação Científica Unicamp - Campinas - SP 19 a 22 de julho de 2015}

\section{REFERÊNCIAS}

COELHO, A. M. Simulação e otimização dos processos de adoçamento e desidratação de gás natural e de refinaria. 2007. 210 f. Dissertação (Mestrado em Engenharia Química) - UNICAMP, Campinas, 2007.

LAWSON, J. D., GARST, A. W. Gas Sweetening Data: Equilibrium solubility os Hydrogen Sulfide and Carbon Dioxide in Aqueous Monoethanolamine and Aqueous Diethanolamine Solutions. Journal of Chemical Engineering Data, v. 21, p. 20-30, 1976.

MENDIETA, L. F. F. Estudo da absorção de $\mathrm{H}_{2} \mathrm{~S}$ e $\mathrm{CO}_{2}$ do gás combustível. 2012. $35 \mathrm{f}$. Trabalho de Conclusão de Curso (Graduação em Engenharia Química) - UFRGS, Rio Grande do Sul, 2011.

TALAVERA, R. M. R. Caracterização de sistemas, simulação e otimização de etapas da planta de processamento de gás natural. 2002. 172 f. Tese (Doutorado em Engenharia Química) - UNICAMP, São Paulo, 2002. 